\title{
PREVENCIÓN DE ALTERACIONES AMBIENTALES QUE INCIDEN EN EL NEURODESARROULODELOS NEONATOS EN LA UCIN: INTERVENCIONES DE ENFERMERIAA ESPECIALIZADA
}

PREVENTION OF ENVIRONMENTAL ALTERATIONS WHICH HAVE AN IMPACT ON THE NEURODEVELOPMENT OF NEONATES AT THE NICU: SPECIALIZED NURSING INTERVENTIONS

Recibido septiembre 2015 Aceptado abril 2016

Correspondencia: Profesora de Carrera Titular "B" Unidad de Investigación de la ENEO. México

Correo lastybals@hotmail.com
Autores:

Dra. Lasty Balseiro Almario Profesora de Carrera Titular "B" Unidad de Investigación de la ENEO. México

E.E.N. Luz María de la Luz Salinas Enfermera Especialista del Neonato. Hospital Materno "Leona Vicario" Bicentenario de Ixtapaluca. Edo. de México

P.E.S.S. Ariadna Lizet Romero Gómez Pasantes del Servicio Social de la Licenciatura en Enfermería y Obstetricia de la ENEO-UNAM. México

P.E.S.S. Giordano Sandro Vázquez Lira Pasantes del Servicio Social de la Licenciatura en Enfermería y Obstetricia de la ENEO-UNAM. México

Palabras clave: Alteraciones ambientales, Enfermería Especializada. Intervenciones, Neonato, Neurodesarrollo.

Keywords: Environmental alterations, Specialized Nursing, Interventions, Neonate, Neurodevelopment. 


\section{RESUMEN}

El objeto de esta revisión fue identificar las principales intervenciones que la Enfermera Especialista del Neonato, debe realizar en el cuidado preventivo de las alteraciones ambientales de la Unidad de Cuidados Intensivos Neonatales (UCIN) en los neonatos fisiológicos y patológicos.

Marco teórico: El medio ambiente de una UCIN está diseñado para sostener médicamente al frágil recién nacido prematuro, pero contrasta ampliamente en el pacífico medio intrauterino, ya que interfiere con el desarrollo de los prematuros en la capacidad de desarrollar respuestas adaptativas a un medio ambiente agresivo para él.

Metodología: Se realizó una búsqueda sistematizada en las bases de datos CINAHL, Medline, Lilacs y Scielo para identificar las alteraciones ambientales de la UCN y con base en este diagnóstico se proponen las intervenciones de enfermería especializada.

Resultados: El personal Especializado de Enfermería cumple una función fundamental en la prevención de las alteraciones ambientales que inciden en el neurodesarrollo de los neonatos en la UCIN. Por ello, se fundamentan y proponen intervenciones especializadas para lograr un cuidado humanizado en los neonatos.

Palabras clave: Alteraciones ambientales, Enfermería Especializada. Intervenciones, Neonato, Neurodesarrollo.

\section{ABSTRACT}

The object of this review was to identify the main interventions which the neonatal specialist nurse must perform on neonates' preventive care during environmental alterations at the NICU.

Theoretical frame: The environment at the NICUs is designed to clinically sustain the frail premature newborn, however; it still contrasts with an optimal healthy intrauterine environment.

Methodology: A systematized database search on CINAHL, Medline, Lilacs and Scielo was carried out to identify data on environmental alterations at the NICU and consequently, propose specialized nursing interventions.

Results: The nursing specialized staff accomplishes fundamental functions to address the environmental alterations which have an impact on the neurodevelopment of neonates at the NICU, and here, specialized interventions offering an optimal neonatal care are proposed.

Key words: Environmental alterations, Specialized Nursing, Interventions, Neonate, Neurodevelopment. 


\section{INTRODUCCIÓN}

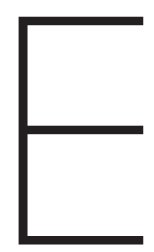

I problema planteado fue identificar cuáles son las intervenciones de enfermería especializada en la prevención de alteraciones ambientales de la UCIN que inciden en el neurodesarrollo de los neonatos en un Hospital Materno Infantil del Estado de México. El objetivo fue identificar las principales intervenciones que la Enfermera Especialista del Neonato debe realizar en el cuidado preventivo de las alteraciones ambientales de la Unidad de Cuidados Intensivos Neonatales (UCIN) en los neonatos fisiológicos y patológicos. Marco Teórico: Según Novoa J.H. y Cols? se denomina neonato crítico a aquel recién nacido enfermo cuya condición patológica afecta uno o más sistemas, que pone en serio riesgo actual o potencial su vida y que presenta condiciones de reversibilidad, que hacen necesaria la aplicación de técnicas de monitorización, vigilancia, manejo y soporte vital avanzado. Por otra parte, la UCIN se define como la sección intrahospitalaria, que garantiza la cobertura asistencial de los neonatos, la asistencia y reanimación en la sala de partos o pabellón e incluye la atención del neonato que se encuentra en puerperio con su madre. Desde el punto de vista operacional se considera el período de atención neonatal desde el momento del nacimiento, hasta las 44 semanas de edad post-concepcional, con un mínimo de 28 días y sin restricciones de peso al nacer. Ahora, para Achury DM² los factores ambientales son el conjunto de todo lo que rodea a la persona en la UCl, por ejemplo, los equipos biomédicos y las condiciones del servicio como la iluminación y el ruido. De igual forma, según Fernández MP.3 el medio ambiente de una UCIN está diseñado para sostener médicamente al frágil recién nacido prematuro y contrasta ampliamente en el pacífico medio ambiente intrauterino. Así, el medio ambiente de la UCIN puede interferir en el desarrollo prematuro, en sus estados conductuales y en la capacidad de desarrollar respuestas adaptativas. Entonces, muchos de los problemas críticos se dan en los sistemas respiratorios y cardiocirculatorio que presentan los neonatos mientras se encuentran en la UCIN, son el resultado de los intentos que ellos realizan para poder adaptarse a este medio ambiente extrauterino y a la agresión que significan la mayoría de los procedimientos médicos. A este aspecto, Sánchez G. y Cols ${ }^{4}$ han argumentado que el ambiente inquietante al que están sometidos los prematuros, que incluyen: ruido intenso relacionado a la actividad de monitores, ventiladores, equipos de succión, teléfonos, alarmas, voces de personas, crea una sobrecarga de estímulos. Estos altos e inapropiados patrones de entrada sensorial pueden alterar las funciones del prematuro y la organización de su conducta. De igual forma, para Zamberian NE y Cols ${ }^{5}$ el ambiente neonatal se caracteriza, frecuentemente, por ser excesivamente estimulante, pudiendo comprometer la recuperación de los recién nacidos, la capacidad laboral del equipo y la satisfacción de los acompañantes. De hecho, las Unidades Neonatales (UN) presentan niveles sonoros intensos, justificado por la implantación de intervenciones. También para Ginovart G. ${ }^{6}$ la estancia del prematuro en la UN provoca efectos no deseados tanto en el recién nacido, como en su familia, ya que la exposición del niño a un medio hostil como es una UCIN dificulta la organización del cerebro en desarrollo. Es decir, las sensaciones y las emociones vividas durante las etapas más precoces, tienen una gran importancia en el desarrollo futuro. Tanto, que las experiencias inadecuadas y estresantes pueden dejar huella en el proceso de desarrollo y provocar consecuencias a medio y largo plazo. En la evolución de los niños muy prematuros aparecen alteraciones del comportamiento, del aprendizaje, emocionales y dificultades sociales. De igual forma, para Ruíz L.? la estancia del recién nacido en la UN, provoca

Enf Neurol Vol. 15. No. 3 septiembre-diciembre 2016 
efectos indeseables en la familia del recién nacido ya que, los padres sienten un impacto emocional que modifica el proceso de crianza. Lo anterior pone de manifiesto que existe una preocupación por el ambiente físico de los servicios de salud, incluyendo Hospitales y Unidades de Terapia Intensiva apoyada en políticas de salud dirigidas a humanizar el cuidado, enfocado en la filosofía del cuidado del desarrollo de los pacientes, especialmente en UN. Entre las alteraciones ambientales se encuentran: el ruido que para Guillen $F^{8}$ ha sido definido como cualquier sonido que causa molestias e irritación, convirtiéndose en un estímulo desagradable para las personas. El ruido se cuantifica mediante el uso de la unidad de decibelios (dB) que se definen con una escala logarítmica. Así, un aumento de $1 \mathrm{~dB}$ en el nivel de ruido corresponde a un aumento de 10 veces en términos del nivel de ruido real. Para Moreira E. y Cols ${ }^{9}$ los primeros estudios sobre la importancia de ruido en el entorno de la UCIN fueron a la década de 1970, en cifras cercanas a los 120 dB. Ahora, según el Ministerio de Sanidad y Política Social de Madrid¹0, el sentido de la audición del neonato se forma a las 20 semanas, y a las 24 semanas los ruidos pueden afectar su conducta, a la 26, tiene el neonato movimientos de los ojos, en respuesta a sonidos, a la 28 es aparentemente maduro, entre la 27 y 35 adquiere la habilidad para distinguir fonemas y con más de 35 semanas, responde selectivamente a sonidos y reconoce la voz materna. Por el contrario, el feto intraútero recibe los sonidos atenuados entre 20 y 50 dB menos, por el medio líquido en que se encuentra. Los estudios en animales sugieren que las células ciliares cocleares son susceptibles de ser lesionadas por sonidos intensos de baja frecuencia. De igual forma, para Brandán R. y Cols $^{11}$ en 1995 la American Academy of Pediatrics mencionó que la exposición al ruido ambiental en la UCIN puede ocasionar daño coclear y alterar el crecimiento y desarrollo normales, ya que las estructuras auditivas inmaduras pueden ser más susceptibles al daño por la combinación de ruido y otros factores de riesgo. También en 1997, el Commiltee on Environmental Health de la Academia Americana de Pediatría, recomendó un máximo nivel de ruido seguro (nivel de presión sonora continua equivalente NPS Leq) de $45 \mathrm{~dB}(\mathrm{~A})$ de día y $35 \mathrm{~dB}$ (A) de noche; refiere que superar éste máximo puede resultar en numerosos efectos adversos para la salud en los prematuros. Finalmente, para Lara CA. y Cols ${ }^{12}$ a menudo los neonatos son expuestos a fuertes ruidos en los servicios de Neonatología, por un mal estado de conservación de las incubadoras o por un manejo inadecuado del personal asistencia. Este excesivo estímulo auditivo genera respuestas fisiológicas inmediatas: Incremento del ritmo cardíaco, cambios de la frecuencia respiratoria, disminución en la saturación de oxígeno del neonato; y a largo plazo, alteraciones en la audición y el desarrollo. De allí, la importancia de su control. Entonces, la exposición a niveles de ruido extremo en la UCIN puede causar daño sensitivo neural, ya que, induce al estrés y contribuye a los trastornos de procesamiento del lenguaje o auditivo en el neonato pretérmino. Datos adicionales sugieren que la cóclea inmadura puede ser más susceptible al daño, que la madura. Además del posible daño del nervio sensorial, el ruido fuerte podría tener consecuencias fisiológicas en el nuevo recién nacido pretérmino en el estrés, lo que produce alteraciones en los niveles de corticosteroides y cambios del sistema autónomo. En cuanto a los días más ruidosos, los datos muestran que el día con mayor nivel de ruido registrado en el lunes con 58.17 dB, similar a lo descrito por Fajardo y Cols $^{13}$. Estos investigadores registraron el mayor ruido el lunes, comparado con el resto de los días de la semana, con 62.01 dB, lo que se relacionó con mayor número de actividades y con el hecho de que las personas en los días subsecuentes, al ver a una persona medir el ruido, bajaban el tono de

\section{2} MMM 
voz. En cuanto al estrés para Schapira I. y Aspres $W^{14}$ aunque se le considera uno de los males "modernos", el estrés aparece desde que los seres vivientes habitan la tierra. Es un proceso biológico y psicológico que se origina ante exigencias y requerimientos internos o externos al organismo, frente a los cuales el neonato no tiene información para una respuesta acorde y que impulsa un mecanismo de ajuste ante la emergencia. Así, para Gallegos J. y Cols ${ }^{15}$ la UN es un área donde existe mucha estimulación para los neonatos por múltiples factores ambientales, entre los que se encuentra los altavoces, los teléfonos, el funcionamiento del equipo electromédico e incluso la conversación del personal. Esto hace que los niveles estándar de decibeles ( $\mathrm{dB}$ ) recomendados por la Academia Americana de Pediatría (AAP): 60 dB en el día y 35 en la noche, se eleven y alcancen hasta 120 dB de presión sonora. Tal situación repercute en el crecimiento y desarrollo del recién nacido, sobre todo del prematuro que al ser estimulado excesivamente, responde fisiológicamente de diferentes maneras. Así, los estímulos de ruido estresantes producen hipoxemia, bradicardia, aumento de la presión intracraneana, hipertensión arterial, apnea, estrés, conducta desorganizada e inefectiva y no adaptativa, e inestabilidad metabólica, ya que aumentan los requerimientos calóricos a partir de glucosa. También se producen: perturbaciones del sueño, irritabilidad, cansancio, vómito y pérdida de apetito en el neonato, especialmente en el prematuro.En relación con la luminosidad para Melgar A. y Bergón E. ${ }^{16}$ la iluminación es el flujo luminoso que incide sobre una superficie y la luz es la unidad de medida que equivale a la iluminación que incide sobre cada m2 de una superficie y que varía según la distancia del foco. De hecho, la mayoría de las UCIN utilizan luz blanca fluorescente las 24 horas del día. Estudios acerca de la intensidad de la luz en la UCIN han demostrado que el promedio del nivel de intensidad de la luz va en un rango de los 60 a 75 lúmenes. Sin embargo, las normas de la Academia Americana de Pediatría para el cuidado perinatal en 1992, recomiendan que la iluminación en la UCIN debe de ser de 60 lúmenes. De hecho, estudios en animales, niños mayores y adultos, indican efectos deletéreos, producto de un ambiente con iluminación continua fluorescente, que incluyen alteraciones del ritmo biológico y de la función endocrina, efectos físicos y bioquímicos negativos, así como también retardo en el crecimiento. Estos hallazgos han motivado distintas investigaciones que sugieren que la luz continua en las UCIN es perjudicial e induce a estados de privación del sueño y a cambios en los ritmos diurnos del neonato. De igual forma, según Torres J. ${ }^{17}$ los recién nacidos hospitalizados en las UCIN están expuestos de forma permanente a la luz brillante con pocas variaciones entre el día y la noche. La utilización de lámparas, luces fluorescentes y la fototerapia como tratamiento médico en los casos de ictericia, incrementan los niveles de intensidad de la luz con efectos perjudiciales en estos neonatos, especialmente los más prematuros. Así, los neonatos que son ingresados a las salas de hospitalización de Recién Nacidos son expuestos a múltiples factores considerados por sus condiciones, especialmente la prematurez, con agresiones físicas ambientales, siendo los más conocidos el ruido, la luz y ondas magnéticas generadas por los diferentes elementos eléctricos de su alrededor, que pueden afectar su situación clínica y evolución posterior en forma de alteraciones en la frecuencia cardiaca, respiratoria, oxigenación, hormonales y trastornos psicológicos como alteraciones del ritmo del sueño, e irritabilidad. Por otro lado, para Castellanos MA. y Cols ${ }^{18}$ también se ha documentado que un ambiente de luz constante puede contribuir a enfermedades de los ojos en infantes prematuros. Es decir, un ambiente de luz constante produce degeneración retiniana, y como ya se comentó, la retina es la vía principal de entrada de la señal de

Enf Neurol Vol. 15. No. 3 septiembre - diciembre 2016 
sincronización para el sistema circadiano, por lo que si se encuentra bajo luz constante, además de sufrir los efectos directos de ésta, las neuronas del núcleo supraquiasmático serán estimuladas de manera continua. En relación con la luminosidad para Melgar A. y Bergón E. ${ }^{16}$ la iluminación es el flujo luminoso que incide sobre una superficie y la luz es la unidad de medida que equivale a la iluminación que incide sobre cada m2 de una superficie y que varía según la distancia del foco. De hecho, la mayoría de las UCIN utilizan luz blanca fluorescente las 24 horas del día. Estudios acerca de la intensidad de la luz en la UCIN han demostrado que el promedio del nivel de intensidad de la luz va en un rango de los 60 a 75 lúmenes. Sin embargo, las normas de la Academia Americana de Pediatría para el cuidado perinatal en 1992, recomiendan que la iluminación en la UCIN debe de ser de 60 lúmenes. De hecho, estudios en animales, niños mayores y adultos, indican efectos deletéreos, producto de un ambiente con iluminación continua fluorescente, que incluyen alteraciones del ritmo biológico y de la función endocrina, efectos físicos y bioquímicos negativos, así como también retardo en el crecimiento. Estos hallazgos han motivado distintas investigaciones que sugieren que la luz continua en las UCIN es perjudicial e induce a estados de privación del sueño y a cambios en los ritmos diurnos del neonato. De igual forma, según Torres J..$^{17}$ los recién nacidos hospitalizados en las UCIN están expuestos de forma permanente a la luz brillante con pocas variaciones entre el día y la noche. La utilización de lámparas, luces fluorescentes y la fototerapia como tratamiento médico en los casos de ictericia, incrementan los niveles de intensidad de la luz con efectos perjudiciales en estos neonatos, especialmente los más prematuros. Así, los neonatos que son ingresados a las salas de hospitalización de Recién Nacidos son expuestos a múltiples factores considerados por sus condiciones, especialmente la prematurez, con agresiones físicas ambientales, siendo los más conocidos el ruido, la luz y ondas magnéticas generadas por los diferentes elementos eléctricos de su alrededor, que pueden afectar su situación clínica y evolución posterior en forma de alteraciones en la frecuencia cardiaca, respiratoria, oxigenación, hormonales y trastornos psicológicos como alteraciones del ritmo del sueño, e irritabilidad. Por otro lado, para Castellanos MA. y Cols' también se ha documentado que un ambiente de luz constante puede contribuir a enfermedades de los ojos en infantes prematuros. Es decir, un ambiente de luz constante produce degeneración retiniana, y como ya se comentó, la retina es la vía principal de entrada de la señal de sincronización para el sistema circadiano, por lo que si se encuentra bajo luz constante, además de sufrir los efectos directos de ésta, las neuronas del núcleo supraquiasmático serán estimuladas de manera continua. En relación con este punto, se sabe con base en la experimentación, que la luz constante, genera un fenómeno de desincronización interna (arritmia) por lo que algunos investigadores proponen un programa asistencial del desarrollo de cuidados neonatales individualizados, donde se sugiere que, dado que el útero es oscuro, los infantes deben desarrollarse mejor en un ambiente de oscuridad que en uno de luz constante. En cuanto al dolor en los neonatos, los componentes en el sistema del dolor se encuentran desde los receptores sensitivos en la piel, hasta áreas sensitivas de la corteza cerebral. La densidad de las terminaciones nerviosas nociceptivas en la piel del recién nacido, marcación de proteínas específicas producidas por los conos de crecimiento axonal, actividad refleja y campos receptivos de neuronas primarias y el desarrollo de sinapsis entre neuronas aferentes primarias interneuronas en el asta posterior de la médula espinal, indican madurez anatómica y funcional del sistema periférico del dolor durante la vida fetal. Así, el recién nacido posee los componentes anatómicos y funcionales para la

\section{4} IMNVWhy Enf Neurol Vol. 15. No. 3 septiembre - diciembre 2016 
percepción del dolor. Para De Fonseca E. y Cols ${ }^{19}$ recientemente se creía que la mielinización nerviosa incompleta evitaba que el neonato experimentase dolor, excepto en un grupo limitado. Sin embargo, se entiende hoy que las vías del dolor y los centros corticales y subcorticales a la percepción del dolor, son bien desarrollados en éstos neonatos y que los sistemas neuroquímicos asociados a la transmisión del dolor están intactos y funcionales. Así, las alteraciones fisiológicas asociadas al dolor en el neonato incluyen: el aumento de la presión arterial y frecuencia cardíaca durante los procedimientos dolorosos. Otras respuestas que pueden servir como indicaciones para la sensación de dolor en el neonato son: expresión contraída con músculos faciales tensos y frente arrugada; refunfuño intermitente o llanto alto y vigoroso; respiración irregular con atragantamientos o retracción; miembros superiores tensos y mantenidos en la misma posición; estado de alerta, inquieto y sin conseguir dormir. Entonces, en el medio hospitalario al menos el 50\% de los niños internados en la UCIN sufren dolor que no siempre es causado por la patología de ingreso del neonato; en la mayoría de casos, el dolor es provocado por las técnicas que realiza el personal de salud como: extracción de sangre, punciones de talón, aspiración de secreciones orofaríngeas, nasofaríngeas y endotraqueales, colocación de catéteres venosos, sondas nasogástricas, instalación de tubo endotraqueal, punción lumbar, introducción de tubos de tórax, eliminación de cintas adhesivas y aplicación de vacunas. El manejo farmacológico, se dá cuando los procedimientos son más agresivos. Las medidas no farmacológicas deben ir siempre acompañadas por tratamiento farmacológico el cual se puede elegir de acuerdo a la escala analgésica de la OMS, en orden ascendente: dolor leve: no opioide \pm adyuvante, dolor moderado: opioide menor \pm adyuvante y dolor severo: opioides mayores \pm no opioide \pm adyuvante. Dentro de las técnicas no farmacológicas con propiedades analgésicas, se encuentra la sacarosa o glucosa al 30\%; vía oral, ofrecida cuando menos, media hora ántes del procedimiento, cuyas propiedades analgésicas nociceptivas parece estar mediadas por receptores opióides disminuyendo el llanto asociado a punciones de talón o venopunciones. Otra técnica analgésico no farmacológico es la estimulación multisensorial amamantando al niño al pecho de su madre durante la intervención dolorosa, aunque también se recomienda facilitar la posición fetal sosteniendo las extremidades del neonato flexionadas cerca del tronco lo cual reduce la frecuencia cardíaca y disminuye el llanto. La succión no nutritiva, estimulada por el chupete también reduce la respuesta fisiológica al dolor. En relación con los procedimientos, los objetivos del cuidado postural del neonato prematuro son: recogerlo en flexión, estimular la flexión activa del tronco y extremidades, de tal manera que se facilite la actividad mano-boca. Por ello, se debe mantener este grado de flexión utilizando nidos o rollos, que proporcionan límites (contención), y posibiliten mayor autorregulación y capacidad para tranquilizarse, lo que a su vez ayuda en la organización de la conducta. También sirve para conseguir unas cabezas más redondas y conseguir posturas más simétricas facilitando los movimientos antigravitatorios. Además, permite estimular la exploración visual del entorno, favoreciendo la alineación corporal ya que, el recién nacido prematuro no ha tenido la oportunidad de desarrollar la flexión fisiológica que ocurre en el último trimestre de la gestación. Además, posee un escaso tono muscular que le incapacita para vencer la acción de la gravedad, lo que le obliga a adoptar un patrón postural en extensión que le aleja de la línea media de relajación, favoreciendo la retracción de los músculos de espalda y cadera. Todo ello, puede producir deformidades posturales que pueden afectar a su desarrollo psicomotor, a la relación de apego con sus padres y a su propia

Enf Neurol Vol. 15. No. 3 septiembre - diciembre 2016 
autoestima cuando madure. Así, el posicionamiento correcto desempeña un papel muy importante en la eficacia de los cuidados. Entonces, para García P. ${ }^{20}$ la contención del cuerpo es otra medida que incrementa la sensación de seguridad del niño, proporcionando quietud y autocontrol, y mejorando la tolerancia al estrés. Muchos prematuros "viajan" a los lados y al extremo de la incubadora buscando fronteras. Las maniobras de contención y recogimiento, tales como envolverlos, tomarles de los dedos o las manos, cruzarles las manos en la línea media, mientras se les manipula, ayuda a la autorregulación. Así, en la manipulación de rutina o en los procedimientos, no se debe levantar al niño en posición supina, dejándole "planear" y arquearse, pues puede producirle sobresalto, apnea o hiperextensión de la cabeza. La mejor técnica es manipular al niño siempre conservando la flexión, al favorecer la posición de prono o lateral. En relación con la interrupción del sueño, para Velayos J.L. ${ }^{21}$ el sueño, biológicamente, no es una falta total de actividad, sino que se puede considerar como un estado concreto conductual. Para lograr un sueño reparador se debe descansar entre siete y ocho horas sin interrupción, ya que, sin importar la fase donde se encuentre, este debe iniciar nuevamente. Los efectos restauradores de las diferentes fases del sueño dejan ver la importancia y la necesidad de que el individuo pueda completar dicho proceso. En el sueño, para Gonzalo Nazar22 el ronquido corresponde a un ruido respiratorio que se produce durante el sueño por la vibración de las estructuras naso-faríngeas, causada por una resistencia al flujo aéreo en la vía aérea superior. En muchos casos, el ronquido puede ser el principal o único síntoma de la apnea obstructiva del sueño. Sin embargo, la mayoría de los expertos considera que el ronquido simple y la apnea obstructiva del sueño corresponden a una misma patología en diferentes etapas, dado que comparten una misma fisiopatología y que es posible encontrar muchos casos con formas intermedias de la patología, formando un verdadero continuo de diferentes grados de severidad. Es por esto que se suelen agrupar bajo el término de trastornos respiratorios relacionados con el sueño en las apneas centrales, las apneas obstructivas y los síndromes de hipoventilación. Entonces, en el neonato gravemente enfermo, la interrupción de los períodos de sueño supone una agresión importante ya que se ve alterado su descanso fisiológico y psicológico, con el correspondiente período de desorganización que esto conlleva, necesitando tiempo, oxígeno y calorías para recuperar tal situación. Así, las continuas interrupciones del descanso aumentan los períodos hipóxicos, las pausas respiratorias, los períodos de apnea, el consumo de oxígeno e incrementan la presión intracraneana. Finalmente, es cuando a la manipulación para Rodríguez L. y De la Mata l.23 las Técnicas de Mínima Manipulación (TMM) son una forma de minimizar el impacto que tiene el ingreso en una UCIN, especialmente para los recién nacidos muy prematuros. Están enmarcadas dentro de los Cuidados Centrados en el Desarrollo (CCD) que es un sistema de cuidados para el neonato que pretende mejorar su desarrollo a través de intervenciones que le favorezcan a él y su familia, entendiéndolos como una unidad (al igual que el control ambiental). Todos los estímulos internos o externos deben presentarse adecuadamente en frecuencia, duración, intensidad, etc., porque tan nocivo es, para los sistemas funcionales, la hiperestimulación y la estimulación fluctuante, como la estimulación a destiempo o su ausencia, especialmente en el caso de los recién nacidos prematuros.

\section{METODOLOGÍA}

l a variable estudiada fue Alteraciones ambientales de la UCIN. La revisión fue retrospectiva, longitudinal y diagnóstica. La población de estudio fueron 123 artículos relativos a Alteraciones ambientales en la UCIN y la

\section{6} MMN $/ 4 y_{W}$ Enf Neurol Vol. 15. No. 3 septiembre - diciembre 2016 
revisión de la bibliografía se hizo mediante una búsqueda sistematizada de la base de datos CINAHL, Medline, LILIACS, Scielo, Cochrane y Artemisa. Los artículos revisados dieron cuenta de las alteraciones ambientales de la UCIN que inciden en el neurodesarrollo de los neonatos, la interferencia en el desarrollo prematuro de sus estados conductuales y en la incapacidad de los neonatos de desarrollar respuestas adaptativas a un medio ambiente extrauterino que le es agresivo. Esto provoca la "desorganización" de su estado fisiológico. El ambiente inquietante tiene que ver los siguientes indicadores: ruido, estrés, luminosidad, dolor por procedimientos médicos y de enfermería, interrupción del sueño, procedimientos y manipulación. Las propuestas de intervención especializada de Enfermería se generan a partir de cada uno de los indicadores estudiados.

INTERVENCIONES DE ENFERMERÍA ESPECIALIZADA DEL NEONATO

- Minimizar el ruido: Para Hernández ME y Cols²4 el ruido se considera un factor de estrés importante para los recién nacidos y para los profesionales de la UCIN. Se ha comprobado que ambos entornos son considerablemente ruidosos. Al evaluar el entorno acústico, se debe tener en cuenta que la UCIN y el trabajo de la incubadora como un sistema. Un estudio demostró que, en general, los niveles de presión sonora dentro de la incubadora son más altos que en la UCIN. Así, el sonido ambiental en la UCIN cruza parcialmente la pared de acrílico de la cúpula incubadora que, a su vez, produce su propio sonido, que resulta del funcionamiento del motor, las actividades de atención y las manifestaciones propias del niño. Dado que el entorno está cerrado, estos sonidos resuenan contra la pared de cúpula, de manera muy sonora, amplificando el ruido que llega al neonato. Entonces, uno de los aspectos que siempre se deben recordar en la gestión del ruido durante la atención neonatal, es que, en la UCIN, los neonatos están expuestos al ruido de impacto, lo que provoca la desorganización de su estado fisiológico, y que los ruidos continuos, no permiten su recuperación. Los especialistas consideran que el fenómeno de la habituación en los recién nacidos, definida como la capacidad de disminuir las respuestas de comportamiento a los estímulos repetitivos, permite el gasto de energía menor o mayor capacidad para seguir durmiendo. Cuando los estímulos ambientales son muy fuertes y continuos, la habituación se ve comprometida y el niño reacciona a estos estímulos hasta que se agota.

- Detectar y disminuir signos de estrés en el neonato: Las niñas y los niños Recién Nacidos de Pretérmino (RNP) requieren cuidados intensivos especializados para mejorar su salud y salvar sus vidas, y estímulos apropiados para mitigar los daños o iatrogenias. Por eso, se hospitalizan en las UCIN. El ambiente interno de éstas Unidades está a cargo de situaciones estresantes y dañinas para niños y niñas. Los neonatos son inmaduros, vulnerables, frágiles y sensibles a los estímulos agresores: el ruido, iluminación, dolor y manipulaciones constantes (procedimientos y cuidados) y, por el nacimiento prematuro, han perdido la homeostasia que poseían en el útero materno. De hecho, las investigaciones han demostrado que el RNP evolucionan mejor modificando el estrés del ambiente hospitalario. Este se reduce disminuyendo los estímulos externos nocivos, manipulándolos con técnicas especiales y aten diéndolos en forma individualizada, humanizada e idónea.

- Mantener las luces apagadas una hora: Estudios realizados con recién nacidos prematuros indican que éstos niños muestran un retraso en el desarrollo de los ritmos circadianos en comparación con los recién nacidos a término. A este atraso, hay que sumar el efecto que ejercen las condiciones ambientales a las que estos niños suelen quedar

Enf Neurol Vol. 15. No. 3 septiembre - diciembre 2016 
expuestos durante el periodo neonatal, ya que la mayoría de los servicios de perinatología los recién nacidos pretérmino son hospitalizados por períodos prolongados bajo condiciones de iluminación (luz constante o luz tenue constante), lo que implica que no existe una alternancia significativa en las intensidades de iluminación entre una fase de luz y una de oscuridad. Debido a lo anterior, recientemente se ha sugerido que la exposición de los infantes a ciclos de luz/oscuridad favorece el incremento del peso y el crecimiento, intrahospitalario. En la atención a los prematuros, es importante el empleo de luces regulables e individualizadas. Para la exploración de los neonatos, lo más adecuado es utilizar los focos individuales de las incubadoras pero evitando enfocar la cara y proteger la cara del neonato de la luz, por ejemplo, creando una sombra con la manta cobertora. Por ello, se deben intentar que el cuidador no se interponga entre el foco y el niño para que con su movimiento no se alternen momentos de luz y sombra. Además, es necesario cubrir las incubadoras con mantas gruesas, pero dejando una pequeña franja para poder visualizar y valorar al niño periódicamente sin necesidad de levantar la manta. Así, para Rodríguez L. y De la Mata Inés ${ }^{25}$ cubrir las paredes de la incubadora con un cobertor permite disminuir la exposición a la luz y disminuir pérdidas por radiación. Entonces, los prematuros no deberían exponerse a la luz directa y en las manipulaciones en las que se precise alta intensidad lumínica, se le deben tapar los ojos del neonato.

- Evitar el dolor en el neonato: Los pretérminos se ven sometidos a múltiples procedimientos dolorosos para resolver sus problemas de salud. El dolor no tratado en el RN contribuye en la morbilidad y mortalidad. Además, la ausencia de dolor es un derecho de todos. Las vías ascendentes que conducen la percepción del dolor, se desarrollan alrededor de la 20 a semana de gestación; y las vías descendentes que inhiben los impulsos dolorosos entrantes, no maduran hasta el último trimestre de gestación, lo que significa que, probablemente, la sensibilidad al dolor de los prematuros sea elevada. Así, para Serrano L. y Cols ${ }^{26}$ se debe valorar el dolor en el neonato con la utilización de Escalas de dolor (PIPP, CRIES, Susan Givens, Bells, NIPS etc...) en las UCIN. Esto permite valorar y registrar el dolor, y aplicar medidas para su tratamiento. También los estímulos dolorosos repetitivos pueden provocar cambios y alteraciones hemodinámicas, respiratorias y del desarrollo cerebral. El llanto derivado del dolor, indica un elevado nivel de excitación si es prolongado o frecuente. Esto aumenta hasta 200\% la demanda energética, eleva el metabolismo basal, disminuye el retorno venoso de la vena cava inferior y reduce la oxigenación. Lo cierto es que los recién nacidos son sometidos a una serie de procedimientos e intervenciones terapéuticas que ocasionan dolor, dado que algunos niños nacen con prematurez extrema, por lo que requieren en algunas ocasiones, hasta de dos meses, de manejo ventilatorio y procedimientos como colocación de catéteres percutáneos, acceso vascular, succión de secreciones, además del constante retiro de cintas adhesivas que lastiman la piel. Es decir, los neonatos están expuestos hasta tres procedimientos dolorosos diariamente. La valoración y tratamiento del dolor neonatal debe ser multidisciplinario, a cargo del médico, enfermeras y familiares, por lo que además del tratamiento farmacológico, es de gran utilidad el uso de técnicas no farmacológicas.

- Estandarizar la colocación de nidos a los recién nacidos: El recién nacido prematuro no ha tenido la oportunidad de desarrollar la flexión fisiológica que ocurre en el último trimestre de la gestación, como respuesta al menor espacio dentro del útero y a un proceso activo de neurodesarrollo. Además, puede precisar una inmovilización prolongada sobre una superficie dura, con el efecto de la gravedad,

\section{8}

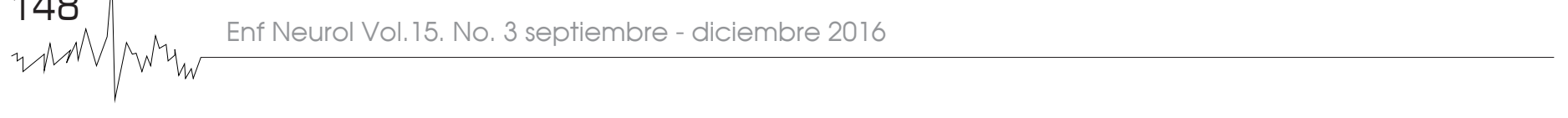


lo que puede producir deformidades posturales como: abducción y rotación de los hombros, mayor hiperextensión cervical con elevación de los hombros y un aplanamiento progresivo de la cabeza, que puede afectar su desarrollo psicomotor a la relación de apego con sus padres y a su propia autoestima cuando el niño madure. Por lo anterior los "nidos" facilitan la contención física, la promoción del estado de sueño, la posición antirreflujo si es necesaria, protegen de pérdida calórica, y son dúctiles en su uso. Las posturas adecuadas del RN, facilitador de la línea media, están asociadas a mayor estabilidad fisiológica y mejoran su desarrollo neuroconductual. Una de las medidas necesarias para evitar el desarrollo de hemorragia intracraneana o su extensión, en el caso de existir, es conservar en los recién nacidos el retorno venoso de la cabeza sin obstrucción. Por ello, posicionar la cabeza del RN en la línea media o hacia un lado, son prácticas que a diario deben priorizarse. También los RN tratados con bloqueadores neuromusculares deben recibir posicionamiento adecuado, a fin de conservar su estabilidad fisiológica, al igual que los RNPT con hipotonía global. Entonces, el posicionamiento adecuado del neonato ayuda a prevenir deformaciones posturales como la abducción y rotación externa de la cadera, la hiperextensión del cuello, la elevación de hombros y el amoldamiento craneal. Con los nidos, los neonatos adoptan posiciones cómodas, confortables, acogedoras (autorregulado). Además, se promueven las termorregulaciones, gastan menor energía en la búsqueda de protección y apoyo y ganan más rápido el peso.

- Minimizar las interrupciones del sueño: Para Shapira I. y Cols ${ }^{27}$ el recién nacido de término y aún más el recién nacido pretérmino, pasan gran parte del día durmiendo. Es lo normal. Cuando no duermen y lloran, se encuentran en "estado de alerta". Este es el momento en el que es conveniente realizar las manipulaciones e intervenciones necesarias, pues el neonato puede interactuar con el medio, sus padres y cuidadores. Así, el estado de alerta o aproximación implica: ojos abiertos, fijación de la mirada, succión activa, movimientos de miembros y respiración más frecuente. Por el contrario, el estado del sueño o retraimiento implica: ojos cerrados, hipotonía muscular, sonrisas, sobresaltos, apneas cortas, movimientos incoordinados de succión, respiración más lenta, y es el momento en que el recién nacido pre término ordena su Sistema Nervioso Central (SNC) y crece y no se debe interferir con procedimientos innecesariamente. La organización del sueño-vigilia, auxilia al recién nacido pre término y término a estructurar, mantener y facilitar los cambios de conciencia evitando los picos de extenuación y agitación. Favorece además, la auto- organización, y la ganancia de peso. Entonces, el ambiente calmado, regular y esquematizado, facilita establecer el patrón repetitivo y de transición gradual. La UN es un área donde existe mucha estimulación para los neonatos por múltiples factores ambientales, entre los que se encuentran los altavoces, los teléfonos, el funcionamiento del equipo electromédico e incluso la conversación del personal. Esto hace que los niveles estándar de dB recomendados por la AAP: 60 dB en el día y 35 en la noche, se eleven y alcancen hasta 120dB de presión sonora 1,2. Tal situación repercute en el crecimiento y desarrollo del recién nacido, sobre todo del prematuro al ser estimulado excesivamente, a lo cual responde fisiológicamente de diferentes maneras. Los estímulos de ruido producen: hipoxemia, bradicardia, aumento de la presión intracraneana, hipertensión arterial, apnea, estrés, conducta desorganizada e inefectiva y no adaptativa, inestabilidad metabólica, ya que aumentan los requerimientos calóricos a partir de la glucosa. Además, se producen perturbaciones del sueño, irritabilidad, cansancio, vómito y pérdida de apetito en el neonato, especialmente en el prematuro.

Enf Neurol Vol. 15. No. 3 septiembre - diciembre 2016 
- Medir constantes vitales en la penumbra: Para Videla ML. ${ }^{28}$ los signos vitales son la determinación de la frecuencia cardíaca, la frecuencia respiratoria y la temperatura corporal. También incluye la presión arterial. El control de las variables vitales es parte de la valoración clínica de Enfermería y generalmente se efectúa acompañada de un monitoreo multiparamétrico en forma permanente, permitiendo evaluar y comparar el valor numérico, la onda de pulso, el trazo electrocardiográfico y el patrón respiratorio con los datos obtenidos. El monitoreo, en algunos casos, permite no realizar contacto con el paciente, principalmente para respetar el descanso y el sueño, pero este no reemplaza al control manual una vez por turno. Esta técnica no solo se realiza en la UCIN, también se lleva a cabo en sala de partos, en la recepción del recién nacido, en la internación conjunta, durante un traslado, en el ingreso a la guardia, en el consultorio y también en el hogar. La medición de las constantes vitales favorece la relación enfermera-paciente durante el procedimiento evitando convertir un "procedimiento de rutina" a las manifestaciones que se pueden percibir y medir en un organismo vivo de forma constante proporcionando información de órganos vitales como cerebro, corazón y pulmón. También, es de suma importancia, mantener limpia la incubadora, lavar y limpiar el habitáculo acrílico cada 24 horas, y siempre que se derrame alimento, sangre, orina y/o heces; pues las condiciones de calefacción son propicias para el crecimiento de gérmenes y microorganismos. Así, la limpieza proporciona visibilidad del recién nacido y lo aísla del resto del ambiente. Por lo tanto, es fundamental conocer su cuidado y manejo.

- Fomentar la lactancia materna y el programa "Mamá Canguro": La leche materna es el mejor alimento para el recién nacido. El momento de lactar en sí es un acto íntimo entre madre e hijo y contribuye de manera importante a la creación del vínculo entre los dos. Además, los niños alimentados con leche materna están más protegidos frente a las infecciones nosocomiales y se ha demostrado que mejoran el cociente de desarrollo y disminución de las tasas de reingreso. Los recién nacidos pretérmino muchas de las veces se ven excluídos de los beneficios de la leche materna al verse fuera del seno materno de manera prematura no dando tiempo a veces a realizar la educación sanitaria a la familia para explicar técnicas de almacenamiento de dicha leche para su posterior administración cuando la situación de salud lo permita. El recién nacido cuando nace necesita encontrarse con su madre, que es el entorno más seguro para él. El contacto piel a piel y la lactancia materna representa el estado normal que permite la óptima adaptación del neonato al medio extrauterino. El contacto piel con piel mejora en el recién nacido el ritmo cardiaco, regula la temperatura, los niveles de glucosa en sangre, el sistema inmunitario, el sueño, la ganancia de peso y la maduración cerebral. Otro punto importante es el sentimiento que despiertan en los padres, el apego con su hijo. Entre las metodologías surgidas para favorecer el neurodesarrollo, cabe destacar la técnica planteada por Rey y Martínez de "madre canguro", en Colombia. Esta alternativa de cuidado diferente al convencional fue y es aplicada a neonatos menores de 2500 gr., con limitaciones en la asistencia de la salud lo que facilita un estado fisiológico, mejora el desarrollo madurativo y el crecimiento emocional del recién nacido. De igual forma, el Programa tiene un impacto positivo sobre la relación madre-hijo, con mejor regulación térmica, sin aumentar el consumo de oxigenación del neonato, menor número de apneas, estabiliza la saturación de oxígeno y la FC, estimula la lactancia y aumenta la secreción de prolactina y de inmunoglobulina A en la leche materna. Además, el Programa aumenta la confianza de los padres en el cuidado de su hijo, disminuye el riesgo de infecciones intrahospitalarias,

\footnotetext{
150 MMN Why Enf Neurol Vol. 15. No. 3 septiembre - diciembre 2016
} 
mejora la orientación neurológica, hay mayor aumento de peso en el recién nacido y es un estímulo neurosensorial apropiado para el recién nacido pretérmino.

\section{CONCLUSIONES}

- El medio intrauterino es líquido, caliente, oscuro, con movimientos rítmicos, con sonidos monótonos y amortiguados, que proporciona el sostén adecuado para que el niño vaya adquiriendo la posición de flexión. En cambio las UCIN en general, mantienen el ambiente ruidoso, muy iluminado, con continuas interrupciones del sueño, intervenciones dolorosas, superficie de apoyo en las cunas duras, y la fuerza de gravedad hace que el niño tienda a mantener una postura de extensión. Evidentemente, los neonatos y más aún los prematuros, no están preparados para responder de manera "organizada" ante tantas agresiones.

- El medio ambiente de las Unidades de Cuidados Intensivos interrumpen el desarrollo de los estados conductuales en los recién nacidos prematuros. Estos estados del neonato que son los ciclos de sueño- vigilia que incluyen: sueño tranquilo, sueño activo, somnolencia, alerta tranquilo, alerta activo y llanto, deben estar organizados y éstos estados, están determinados por factores endógenos e influencias del medio ambiente. Por lo anterior, conviene evitar en el neonato grave, la interacción en el medio ambiente, colocándolo lejos de la agresión para incidir positivamente en su neurodesarrollo

- Los neonatos también manifiestan dolor. Por ello, la calidez humana, el manejo gentil, las caricias, el arrullo, la música suave, envolverlo en una manta suave, hablarle con un tono tranquilo y minimizar la estimulación nociva del personal, son procedimientos favorables para disminuir el dolor en el neonato. También se debe intentar que el ambiente sea lo más agradable posible al disminuir la luz intensa, el ruido y agrupar los procedimientos. Es necesario tomar en cuenta todos estos aspectos, para disminuir el dolor intenso en los neonatos.

- Las enfermeras y los médicos que atienden a los neonatos deben aplicar programas individualizados de mínima manipulación, intentando que la mayoría de las exploraciones, tomas de muestras e intervenciones de otro tipo, coincidan en el tiempo de "alerta". Estas simples medidas podrían facilitar la estabilización del paciente grave y ayudar al desarrollo del niño. Además, son muy necesarios los programas de capacitación para proponer a los profesionales de la salud para que reconozcan y practiquen las estrategias y actividades que facilitan el desarrollo sensoriomotor de los neonatos y hacerles menos agresiva su estancia en la UCIN. 


\section{REFERENCIAS BIBLIOGRÁFICAS}

1. Novoa JM, Milad M, Vivanco S, Fabres J. y Ramírez R. Recomendaciones de organización, características y funcionamiento en Servicios o Unidades de Neonatología Chilena de Pediatría. Santiago de Chile. Marzo-Abril, 2009; 80 (2): 166-787. Disponible en: http://umw.scie lo.cl/podf/rco//80n2/art10,poff Consultado el día 1 de marzo del 2015.

2. Achury DM., Delgado A. y Ruíz M. El ruido y las actividades de enfermería: factores perturbadores del sueño. Enfermería: imagen y desarrollo. Bogotá, Enero- Abril, 2013; (15) 1: 57- 63. Disponible en hitpo://revistas.javeriana.edu.co/index.pho/imagenydesarrollo/article/ viewFile/6025/4868 Consultado el día 21 de Marzo de 2175

3. Fernández M.P. Intervención sensorio-motriz en recién nacidos prematuros. Pediatría Electrónica. Santiago de Chile, Octubre, 2004; 1 (1): 1-20. Disponible en: hitp//:wnw.revistape diatria.cl/vol7num7/pdf/intervención.pdf. Consultado el día 19 de Marzo del 2015.

4. Sánchez G., Rodríguez I., Quintero L., Nieto G., Centro D., Zapata A. Comparación de los niveles de decibeles (ruido) en las áreas de atención Neonatales. Medicina Universitaria México, Julio-Sept, 2012; 14 (56): 1-7 Disponible en: http://apps.elsevier.es/watermark/ctlservlet? $f=10 \&$ pident_articulo=90167660\&pident_usuario=0\&pcontactid=\&pident_revista=304\&-

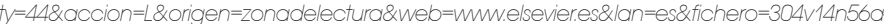
90167660,00ł001.paff Consultado el día 12 de Marzo de 2015.

5. Zamberian N.E., Ine C, Venderley J., Mara L.M., Fortuna C.M. y Silván C.G. Impacto de un programa participativo de reducción de ruido en una Unidad Neonatal. Enfermagen. Riberao Preto, Enero-Febrero, 2012; 20 (1): 1-8- Disponible en:http://mun.scielo.br/pdf/rlae/ V20n1/es_15pdf Consultado el día 20 de marzo de 2015

6. Ginovart G. Cuidados centrados en el desarrollo: un proyecto común Psicología, Aloma Barcelona. 2010.26: 15-27. Disponible en htto:/ / wuw raco cot/index.pho/Aloma/article/viewFile/196127/262943 Consultado el 17 de marzo 2015

7. Ruiz A, Rodríquez R., Mira M..., Robles C., Jerez A, González A, Fernández MS., Sánchez A., Ruiz M., Piñar R., Ortega M.J., Muñoz T y Rodríguez MS. Cuidados Neonatales Centrados en e desarrollo. Boletín SPAO. Granada, España, 2013; 7 (2): 39-57

8. Guillen F. Bernal M. García S. García M. J. Rosario C. Álvarez M. C. Martínez M. Piña L. M Calidad del sueño en los pacientes ingresados en UCl: relación con estresores ambientales Enfermería Docente. Madrid, 2013: 100: 34-39.

9. Moreira E., Guisburg R., De Araujo MA y Yoshiko. Ruido a la Unidad de Cuidados Intensivos Neonatales y dentro de la Incubadora. Enfermagen. Riberao Preto, Sao Paulo, Sept-Dic, 2011 19 (5) 3 Disponible en: htto:muw2.unifesp.br/acta/artigo.pho?volume=20\&ano=2007\&nume ro=4\&item $=3$ Consultado el día 8 de marzo del 2015

10. Ministerio de Sanidad y Política Social. Disminución del ruido y adecuación del nivel de luz en la Unidad de Cuidados Intensivos Neonatales. Los cuidados desde el nacimiento Recomendaciones basadas en pruebas y nuevas prácticas. Ed. Ministerio de Sanidad y Politica Social. Centro de publicaciones. Madrid, 2010:35-45 Disponible en: htto:/ / mum.msss gob.es/organizacion/sns/planCalidadSNS/pdf/equidad/cuidadosDesdeNacimiento.pdf Consultado el 4 de marzo del 2015

11. Brandán R., Halloy N., Sánchez MA., Sánchez LD., Sueldes J., Rocha LA, Herrera M., Inés V. y Olivera JM. Contaminación acústica en salas de Neonatología. Dpto. de Bioingeniería Buenos Aires, 2010: 1 Disponible en: http://muw.theko.com.uy/docs/doc_002.pdf Consultado el día 13 de marzo del 2015

12. Lara C. A, Alvarado R., Luna L. y Vilcahuamán L. Evaluación del ruido en las incubadoras del servicio de Neonatología del Instituto Especializado Perinatal IEMP de Lima, Perú. Facultad de Ciencias e Ingenieŕa. Pontificia Universidad Católica de Perú. Neonatología. Lima, 2007: 69-99

13. Fajardo D.L., Yurany S. y Argote L. A. Niveles de ruido en la Unidad de Cuidados Intensivos Neonatales "CIRENA" del Hospital Universitario del Valle en Calli. Colombia Médica. Bogoté 2007: 38Suplen Z: 64- 71. Disponible en: http//:colombiamedica.univalle.edu.co/index.pho/ comedica/article/view/538/958+\&cd=1\&h/=es-419\&ct=clnk\&gl=mx. Consultado el día 13 de Marzo del 2075

14. Schapira I. y Aspres N. Estrés en recién nacidos intemados en la Unidad de Cuidados Intensivos (UCIN): propuestas para minimizar sus efectos. Hospital Materno Infantil Ramón Serdán. Buenos Aires, 2004; 23 (3): 113-121 Disponible en: http:j/ muw.fundasamin.org.ar/archivos/Revista de Enfermeria_07.pdf\%20modificada.pdf Consultado el día 8 de marzo de 2015
15. Gallegos J. Reves J. Fernández VA y González L.O. Índice de ruido en la Unidad Neonatal. Su impacto en el recién nacido. Acta Pediátrica de México. México, Enero, Febrero; 2017 32 (1): 5- 74. Consultado el día 26 de Marzo del 2015

16. Melgar A y Bergon E. Protocolo de luz y ruido. Ed. Hospital 12 de Octubre. Servicio de Neonatología. Madrid, 2017: 7-6.

17. Torres J. Efectos medioambientales de la Unidad de Cuidados Intensivos en los Reciér Nacidos. Gastrohnup. Bogotá, Enero-Abril, 2014; 16 (1): $11-17$ Disponible en: hitp://evgastrohnup.univalle.edu.co/a14v16n1/a14v16n1art2.pdf+\&h/=es\&ct=clnk\&gl=es Consultado el día 15 de marzo del 2015

18. Castellanos M.A., Vásquez S., Palma M, Ubaldo L., Cervantes S., Rojas A. y Escobar C. Desarrollo de los ritmos biológicos en el recién nacido. Cátedra Especial Dr. Ignacio Chávez Facultad de Medicina. México, Mayo- Junio, 2013; 56 (3): 1- 27. Disponible en: http://mm scielo.org.mx/scielo.pho?pid=S002617422013000700005\&script=sci_arttext Consultado el díd 25 de Marzo del 2075

19. De Fonseca E. Lima I., Cardoso F. v Beresford H. El estrés en el neonato prematuro: una reflexión axiológica acerca de posible influencia de los factores sensitivos ambientales en una Unidad de Terapia Intensiva Neonatal. Fitness Performance. Rio de Janeiro, Sept-Oct. 2008: 7 (5):349

20. García P. Cuidado neonatal con atención al desarrollo. Especializada de Pediatría. Madria 2002 58; (1): 28-36 Disponible en: http//sicapacitacion.com/libmedicos/Cuidados\%20Neonatal.pdf Consultado el día 16 de marzo del 2015.

21. Velayos J.L. Moleres FJ. Irujo AM. DYllanes F. y Patemain B. Bases anátomicas del sueño Anales del Sistema Santuario de Navarra. Madrid, 2007: 30 (1): 7-15. Disponible en: http:/ scielo.iscili.es/scielo.php?script=sci anttext\&pid=S1137-66272007000200002 Consultado el día 2 de marzo del 2074

22. Nazar G. Trastornos Respiratorios del sueño en la edad pediátrica. Médica de la Clínica los Andes. Madrid. 2013: 24 (3): 403-411. Disponible en htto://aposelsevieres/watermark/ ctl_serviet?_f $=10 \&$ pident_articulo=90360827\&pident_usuario=0\&pcontactid=\&pident_re

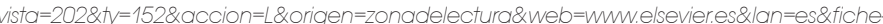
ro=202v24n03a90360827pdf001.pdf Consultado el día 31 de marzo del 2015

23. Rodríguez $L$ y De la Mata l. Procedimiento de mínima manipulación en el neonato Enfermería Castilla León. Madrid. 2014: 6 (2): 58-64 Disponible en: htto:/ muwrevistoenenfer meriacyl.com/index.php/revistaenfermeriacy//issue/current/showToc Consultado el día 4 de marzo del 2014

24. Hernández M.E. y Ocampo A. M. Evaluación de protocolo para minimizar el estrés en neonatos con peso al nacer menor a 2.500 gramos en el Hospital Dr. Carlos Sáenz Herrerd Enfermería en Costa Rica Costa Rica. 2009: 29 (2): 5- 11. Disponible en htto:// revistaenfermeria cr/ediciones/enfermeriaen-costarioatvolumen 3122008. Consultado el día 18 de Marzo del 2015

25. Rodríquez L.y De la Mota l. Procedimiento de control ambiental en Neonatología. Biblioteca Las Casas. Fundación Índex. Granada, España, 2013: 9 (1): 1-13 Disponible en: htlp://mm index_f.com/lascasas/documental//c0867.php. Consultado el día 30 de marzo del 2015.

26. Serrano L., Muñoz E. Importancia de la aplicación de los cuidados centrados en e desarrollo en las Unidades Neonatales y su repercusión en el bienestar de la familia Fundación Índex. Granada, España, 2011; V (14): 8 Disponible en: http://index-f.com/para/ n14/155d.php Consultado el día 17 de marzo del 2015.

27. Shapira I.T. Parareda V., Coria M.B. y Roy E. Propuestas de intervención ambiental y en e desarrollo de recién nacidos de alto riesgo. Hospital Matemo Infantil Ramón Serdán: Santiago de Chile, 1994 XIII (3): 107-109.

28. Videla ML Revisando Técnicas: Control de signos vitales. Enfermería Neonatal. Fundación UNICEF, Buenos Aires, Sept. 2010; 3 (10): 5. Disponible en: http:/ muw.fundasamin.org.ar/archvos/Revisando\%20T\%C3\%A9cnicas\%20\%20Control\%20de\%20signos\%20vitales.pdf Consultado el día 15 de marzo del 2015

152

MMNMYh Enf Neurol Vol.15. No. 3 septiembre - diciembre 2016 